04,05

\title{
Квантовая теория пьезоэлектрического эффекта в редкоземельных ферроборатах
}

\author{
(С) А.И. Попов ${ }^{1,4}$, Д.И. Плохов ${ }^{2,3}$, А.К. Звездин ${ }^{2,5}$ \\ ${ }^{1}$ Национальный исследовательский университет „Московский институт электронной техники“, \\ Москва, Зеленоград, Россия \\ ${ }^{2}$ Институт общей фризики им. А.М. Прохорова РАН, \\ Москва, Россия \\ ${ }^{3}$ Российский университет дружбы народов, \\ Москва, Россия \\ ${ }^{4}$ Московский фризико-технический институт (национальный исследовательский университет), \\ Долгопрудный, Россия \\ ${ }^{5}$ Физический институт им. П.Н. Лебедева РАН, \\ Москва, Россия \\ E-mail: dmitry.plokhov@gmail.com, zvezdin.ak@phystech.edu
}

Поступила в Редакцию 19 ноября 2020 г.

В окончательной редакции 19 ноября 2020 г.

Принята к публикации 20 ноября 2020 г.

Развита квантовая теория пьезоэлектрического эффекта в редкоземельных ферроборатах. Установлено, что за возникновение эффекта отвечают преимущественно одноионные механизмы: при наличии механических напряжений в кристалле индуцируются дипольные электрические моменты непосредственно в электронных $4 f$-оболочках редкоземельных ионов, что приводит к поляризации кристалла. Количественно эффект рассмотрен на примере ферроборатов неодима и самария, в частности, в интервале от 0 до $300 \mathrm{~K}$ получены температурные зависимости пьезоэлектрических модулей. Дополнительно показано, что при температурах ниже точки Нееля при деформации кристалла в магнитном поле также возможно возникновение поляризованности кристалла вдоль кристаллографической оси $c$. Рассчитаны температурные, полевые и ориентационные зависимости магнитоиндуцированных пьезомодулей.

Ключевые слова: пьезоэлектрический эффект, ферробораты, редкоземельные ионы, кристаллическое поле.

DOI: 10.21883/FTT.2021.03.50592.241

\section{1. Введение}

Как хорошо известно, (прямой) пьезоэлектрический эффект заключается в том, что диэлектрик поляризуется под действием механического напряжения. Напротив, под действием приложенного внешнего электрического поля диэлектрический образец деформируется (обратный пьезоэлектрический эффект). Прямой эффект был открыт Ж. и П. Кюри в 1880 г. [1], а, исходя из общих термодинамических соображений, Липпман [2] в 1881 г. предсказал существование обратного эффекта, который в том же году был экспериментально обнаружен братьями Кюри [3].

Пьезоэлектрический эффект является линейным: деформирование пьезоэлектрика сопровождается появлением в нем объемного электрического дипольного момента, пропорционального величине деформации; внутренние напряжения, возникающие в пьезоэлектрике во внешнем электрическом поле, также пропорциональны первой степени напряженности поля. Необходимым условием возникновения пьезоэлектрического эффекта является отсутствие в кристалле центра симметрии [4]. Из 32-ух кристаллических классов допускают пьезоэлектричество всего лишь 20, в частности класс $D_{3}$ ромбоэдрической системы, к которому от- носятся рассматриваемые в настоящей работе ферробораты.

Пьезоэлектрические материалы находят множество практических применений [5]. Мировой рынок пьезоэлектрических устройств оценивается более, чем в 20 млрд.долларов США с тенденцией к постоянному росту [6]. Среди наиболее важных устройств следует упомянуть следующие: пьезоэлектрические датчики и приводы [7], преобразователи [8], электрические генераторы [9], двигатели вращения [10] и др. Пьезоэлектрический эффект также формирует основу для ряда научных инструментальных методов с атомным разрешением [11]: сканирующая туннельная микроскопия (СТМ), атомно-силовая микроскопия (ACM), магнитносиловая микроскопия (МСМ) и др.

Традиционные термодинамические теории, описывающие пьезоэлектрический эффект, по большей части являются феноменологическими, базирующимися на анализе симметрии кристалла; они могут весьма просто предсказывать наличие (или отсутствие) эффекта, но их существенным недостатком является невозможность получения в рамках таких теорий точных количественных температурных и полевых зависимостей.

В течение последних десятилетий развивается иной подход, основанный на вычислениях $a b$ initio, из пер- 
вых принципов, не требующий использования какихлибо существенных экспериментальных данных $[12,13]$. Большинство первопринципных расчетов для пьезоэлектриков основаны на теории функционала плотности (DFT), в свою очередь, большинство из них выполняются в приближении локальной плотности (LDA). Расчеты $a b$ initio заложили основу для базового понимания пьезоэлектрических свойств чрезвычайно употребительных в настоящее время керамик на основе цирконата-титаната свинца (ЦТС) [14]. По мере развития теории и мощностей вычислительной техники постепенно расширяется диапазон изучаемых свойств, увеличивается точность предсказаний. В вычислительном отношении уже становится возможным проектировать теоретическими методами материалы с требуемыми свойствами.

Главным недостатком керамик ЦТС является то, что они содержат свинец. Европарламентом принят закон [15], ограничивающий использование в электротехнике соединений свинца, кадмия, ртути и ряда других вредных и опасных веществ, но он не затрагивает материалы, используемые в пьезотехнике. Это обусловлено тем, что активный поиск веществ, способных во всех отношениях заменить систему ЦТС, пока не увенчался успехом. Несмотря на значительные усилия в деле разработки замещающих материалов, предпринятые за последние два десятилетия, эта проблема не является окончательно решенной. Не содержащая свинец сегнетоэлектрическая керамика не имеет всех тех свойств, которые делают систему ЦТС незаменимой для практических применений. Задача о поиске и исследовании новых пьезоэлектрических материалов по-прежнему остается актуальной.

В настоящей работе предлагается обратить внимание на редкоземельные ферробораты как на новые нетипичные пьезоэлектрические материалы. В последние годы эти соединения привлекают к себе все большее внимание. Они являются (несобственными) мультиферроиками, обладающими большой величиной магнитоэлектрического эффекта, что, помимо число фундаментального интереса, представляет и практический интерес, возникающий вследствие возможности управления магнитным состоянием вещества с помощью электрического поля и наоборот [16].

Преобладающий вклад в формирование магнитных и магнитоэлектрических свойств и характеристик этих соединений дают редкоземельные $f$-ионы. Этот вывод подтверждается малой величиной магнитоэлектрического эффекта в ферроборате иттрия [17], а также его наличием в родственных редкоземельных алюмоборатах $[18,19]$. Для редкоземельных ионов характерно слабое обменное $f-f$ взаимодействие, они обладают значительным орбитальным моментом, что при наличии низко-симметричного кристаллического поля в условиях „замораживания“ магнитных моментов приводит к доминированию одноионных механизмов в возникновении явлений в содержащих редкоземельные ионы материалах, в отличие от межионных механизмов, характерных для соединений $d$-элементов.

Основным проявлением магнитоэлектрического явления в редкоземельных ферроборатах является индуцирование магнитным полем электрической поляризации кристалла, что было исследовано в работе [20]. В настоящей работе рассматривается возникновение электрической поляризации кристаллов ферроборатов при их деформации. Задачей исследования служит установление механизма и количественное описание этого явления.

\section{2. Структура редкоземельных ферроборатов}

Кристаллическая структура ферроборатов $\mathrm{RFe}_{3}\left(\mathrm{BO}_{3}\right)_{4}$, где $\mathrm{R}$ - редкоземельный элемент, описывается пространственной группой симметрии $R 32$ $\left(D_{3}\right)$. Группа $D_{3}$ содержит ось симметрии 3-го порядка, совпадающую с кристаллографической осью $c$, а также лежащие в перпендикулярной ей базовой плоскости $a b$ три оси симметрии 2-го порядка, одна из которых совпадает с кристаллографической осью $a$. В настоящей работе будет использоваться такая система координат, в которой оси $z$ и $x$ направлены соответственно вдоль осей $c$ и $a$. Орты координатных осей обозначим $\mathbf{e}_{x}, \mathbf{e}_{y}$ и $\mathbf{e}_{z}$.

Редкоземельные ферробораты содержат две обменно связанные магнитные подсистемы, состоящие из ионов железа $\mathrm{Fe}^{3+}$ и редкоземельных ионов $\mathrm{R}^{3+}$. Величина антиферромагнитного взаимодействия между ионами $\mathrm{Fe}^{3+}$ на порядок больше обменного взаимодействия между ионами $\mathrm{Fe}^{3+}$ и $\mathrm{R}^{3+}$. В свою очередь, обменное взаимодействие между редкоземельными ионами $(\leqslant 1$ K) еще на порядок меньше, и учитываться не будет. Обменное взаимодействие между ионами железа при низких температурах $\left(T<T_{N}\right.$, температура Нееля $\left.T_{N} \sim 30 \ldots 40 \mathrm{~K}\right)$ формирует антиферромагнитное упорядочение двух магнитных подрешеток $\mathbf{M}_{1,2}$ ионов железа. В состоянии магнитного упорядочения ионов железа редкоземельные ионы в ферроборатах разбиваются на две подрешетки, первую из которых составляют ионы, обменно взаимодействующие преимущественно с ионами железа, составляющими подрешетку $\mathbf{M}_{1}$, а вторую - ионы, взаимодействующие с подрешеткой $\mathbf{M}_{2}$. В присутствии внешнего магнитного поля Н на редкоземельные ионы в подрешетках 1 и 2 действуют эффективные магнитные поля

$$
\mathbf{H}_{1,2}=\mathbf{H}+\lambda \mathbf{M}_{1,2},
$$

где $\lambda$ представляет собой константу молекулярного поля $\mathbf{H}_{\mathrm{mol}}=\lambda \mathbf{M}$.

Важнейшим взаимодействием, определяющим свойства редкоземельных соединений, является взаимодействие редкоземельных ионов с окружением, описываемое гамильтонианом кристаллического поля. Взаимодействие редкоземельных ионов с кристаллическим и эффективными магнитными полями формируют основной (невозмущенный) спектр редкоземельных ионов. Поскольку симметрия окружения редкоземельных ионов в 
Таблица 1. Уровни энергии и соответствующие волновые функции основного мультиплета ионов $\mathrm{Nd}^{3+}$ и $\mathrm{Sm}^{3+}$ в кристаллическом поле $(\cos \alpha=0.980, \sin \alpha=0.201)$

\begin{tabular}{c|c|c|c}
\hline Ион & $k$ & $E_{k}, \mathrm{~cm}^{-1}$ & $\left|\psi_{k}\right\rangle,\left|\bar{\psi}_{k}\right\rangle$ \\
\hline $\mathrm{Nd}^{3+}$ & 0 & 0 & $0.498\left| \pm \frac{7}{2}\right\rangle+0.129 i\left| \pm \frac{1}{2}\right\rangle+0.858\left|\mp \frac{5}{2}\right\rangle$ \\
& 1 & 72 & $0.531\left(\left|+\frac{9}{2}\right\rangle \pm\left|-\frac{9}{2}\right\rangle\right) \pm(0.242 \pm 0.399 i)\left(\left|+\frac{3}{2}\right\rangle \pm\left|-\frac{3}{2}\right\rangle\right)$ \\
& 2 & 146 & $0.840\left| \pm \frac{7}{2}\right\rangle+0.174 i\left| \pm \frac{1}{2}\right\rangle-0.514\left|\mp \frac{5}{2}\right\rangle$ \\
& 3 & 229 & $0.467\left(\left|+\frac{9}{2}\right\rangle \pm\left|-\frac{9}{2}\right\rangle\right) \mp(0.276 \pm 0.454 i)\left(\left|+\frac{3}{2}\right\rangle \pm\left|-\frac{3}{2}\right\rangle\right)$ \\
& 4 & 326 & $0.215\left| \pm \frac{7}{2}\right\rangle-0.976 i\left| \pm \frac{1}{2}\right\rangle+0.022\left|\mp \frac{5}{2}\right\rangle$ \\
\hline $\mathrm{Sm}^{3+}$ & 0 & 0 & $i \sin \alpha\left| \pm \frac{5}{2}\right\rangle-\cos \alpha\left|\mp \frac{1}{2}\right\rangle$ \\
& 1 & 194 & $\left| \pm \frac{3}{2}\right\rangle$ \\
& 2 & 247 & $\cos \alpha\left| \pm \frac{5}{2}\right\rangle+i \sin \alpha\left|\mp \frac{1}{2}\right\rangle$
\end{tabular}

ферроборатах описывается группой $D_{3}$, то во введенной выше системе координатат гамильтониан кристаллического поля имеет вид

$$
\begin{aligned}
\mathscr{H}_{\mathrm{CF}}= & B_{0}^{2} C_{0}^{2}+B_{0}^{4} C_{0}^{4}+B_{0}^{6} C_{0}^{6}+i B_{3}^{4}\left(C_{3}^{4}+C_{-3}^{4}\right) \\
& +i B_{3}^{6}\left(C_{3}^{6}+C_{-3}^{6}\right)+B_{6}^{6}\left(C_{6}^{6}+C_{-6}^{6}\right) .
\end{aligned}
$$

Здесь $C_{q}^{k}=\sum_{i=1}^{n} C_{q}^{k}(i)$, где $C_{q}^{k}(i)-$ одноэлектронные неприводимые тензорные операторы, действующие на $i$-й электрон в $4 f$-оболочке редкоземельного иона $\left(n-\right.$ общее число электронов в ней), а $B_{q}^{k}-$ вещественные константы кристаллического поля. Числовые значения констант кристаллического поля могут быть рассчитаны по экспериментально получаемым с высоким разрешением оптическим спектрам поглощения [21,22]. В настоящее время константы кристаллического поля, действующего на ионы неодима, определены с весьма высокой степенью надежности: $B_{0}^{2}=551 \mathrm{~cm}^{-1}, B_{0}^{4}=-1239 \mathrm{~cm}^{-1}, B_{0}^{6}=519 \mathrm{~cm}^{-1}$, $B_{3}^{4}=697 \mathrm{~cm}^{-1}, B_{3}^{6}=105 \mathrm{~cm}^{-1}, B_{6}^{6}=339 \mathrm{~cm}^{-1}$. К сожалению, столь же надежного набора параметров кристаллического поля для иона самария в ферроборате на сегодняшний день не существует. В настоящей работе, ввиду сходства между ионами $\mathrm{Sm}^{3+}$ и $\mathrm{Nd}^{3+}$, для расчета спектра иона самария используются константы кристаллического поля иона неодима, приведенные выше. В кристаллическом поле происходит расщепление основного мультиплета редкоземельного иона на крамерсовские дублеты: пять для иона $\mathrm{Nd}^{3+}$ и три для иона $\mathrm{Sm}^{3+}$, см. табл. 1.

\section{3. Пьезоэлектрический эффект в редкоземельных ферроборатах}

Рассмотрим теперь влияние деформации кристалла на редкоземельные ионы. Воздействие деформации на $f$-электроны редкоземельного иона в ферроборате можно описать гамильтонианом (в билинейном по компонентам тензора деформации и координатам электронов приближении)

$$
\mathscr{V}_{d}=\sum_{i=1}^{n} c_{1}\left(\left(u_{x x}-u_{y y}\right) x_{i}-2 u_{x y} y_{i}\right)+c_{2}\left(u_{y z} x_{i}-u_{x z} y_{i}\right)
$$

где $u_{\alpha \beta}$ - компоненты тензора деформации, $x_{i}$ и $y_{i}-$ координаты $i$-го $4 f$-электрона, а $c_{1}$ и $c_{2}-$ некоторые константы.

Приложим теперь к кристаллу внешнее электрическое поле Е. Энергия взаимодействия $f$-электронов редкоземельного иона с этим полем может быть представлена в виде

$$
\mathscr{V}_{e}=-\mathbf{d E}=e \sum_{i=1}^{n} \mathbf{E r}_{i}
$$

Гамильтонианом возмущения задачи анализа возникновения электрической поляризации ферроборатов при деформации является

$$
\mathscr{V}=\mathscr{V}_{d}+\mathscr{V}_{e} .
$$

Актуальные для рассматриваемой задачи линейные по $E$ поправки к уровням энергии редкоземельного иона возникают во втором порядке теории возмущений с малым параметром $\| \mathscr{V} \mid / W \ll 1$, где $\|\mathscr{V}\|-$ норма оператора $\mathscr{V}$, а $W$ - разность энергии возбужденных $4 f^{n-1} 5 d$ и $4 f^{n-1} 5 g$ и основной $4 f^{n}$ конфигураций. Для редкоземельного иона типично $W$ порядка $10^{5} \mathrm{~cm}^{-1}$. Эти поправки имеют вид

$$
\begin{aligned}
E_{g}^{(2)} & =-\sum_{l^{\prime}, e_{l^{\prime}}} \frac{1}{W_{l^{\prime}}} \\
& \times\left(\left\langle g\left|\mathscr{V}_{e}\right| e_{l^{\prime}}\right\rangle\left\langle e_{l^{\prime}}\left|\mathscr{V}_{d}\right| g\right\rangle+\left\langle g\left|\mathscr{V}_{d}\right| e_{l^{\prime}}\right\rangle\left\langle e_{l^{\prime}}\left|\mathscr{V}_{e}\right| g\right\rangle\right),
\end{aligned}
$$

где $|g\rangle$ - состояния иона, принадлежащие основной $l^{n}$ конфигурации $(l-$ символ, обозначающий орбитальное 
квантовое число электронов, для редкоземельных ионов $l=3),\left|e_{l^{\prime}}\right\rangle-$ состояния возбужденной $l^{n-1} l^{\prime}$ конфигурации с $l^{\prime}=l \pm 1$, а $W_{l^{\prime}}$ - разность энергий состояний $\left|e_{l^{\prime}}\right\rangle$ и $|g\rangle$.

Уравнение (6) записано в пренебрежении расщеплением уровней $l^{n-1} l^{\prime}$ конфигурации. К основным уровням ближе расположена конфигурация с $l^{\prime}=l-1$, и в дальнейшем будет учитываться только ее вклад в поправки (6).

Воспользуемся результатами, приведенными в [23], и, исходя из (6), найдем, что взаимодействие деформации с поляризацией может быть описано эффективным гамильтонианом (в расчете на один редкоземельный ион).

$$
\mathscr{H}_{\text {eff }}=A_{1} \mathscr{H}_{1}+A_{2} \mathscr{H}_{2},
$$

где

$$
\begin{aligned}
\mathscr{H}_{1}= & \sqrt{\frac{2}{3}} C_{0}^{2}\left(2 u_{x y} E_{y}-\left(u_{x x}-u_{y y}\right) E_{x}\right) \\
& +\left(C_{-2}^{2}+C_{2}^{2}\right)\left(\left(u_{x x}-u_{y y}\right) E_{x}+2 u_{x y} E_{y}\right) \\
& -i\left(C_{-2}^{2}-C_{2}^{2}\right)\left(2 u_{x y} E_{x}-\left(u_{x x}-u_{y y}\right) E_{y}\right) \\
& +\left(C_{-1}^{2}-C_{1}^{2}\right)\left(u_{x x}-u_{y y}\right) E_{z}-i\left(C_{-1}^{2}+C_{1}^{2}\right) 2 u_{x y} E_{z}, \\
\mathscr{H}_{2}= & \sqrt{\frac{2}{3}} C_{0}^{2}\left(u_{x z} E_{y}-u_{y z} E_{x}\right)+\left(C_{-2}^{2}+C_{2}^{2}\right)\left(u_{y z} E_{x}+u_{x z} E_{y}\right) \\
& -i\left(C_{-2}^{2}-C_{2}^{2}\right)\left(u_{x z} E_{x}-u_{y z} E_{y}\right) \\
& +\left(C_{-1}^{2}-C_{1}^{2}\right) u_{y z} E_{z}-i\left(C_{-1}^{2}+C_{1}^{2}\right) u_{x z} E_{z}, \\
& A_{1,2}=-e c_{1,2} \sqrt{\frac{27}{70}}\left(\frac{r_{f d}^{2}}{W_{d}}+\frac{5 r_{f g}^{2}}{9 W_{g}}\right) .
\end{aligned}
$$

Пьезоэлектрический билинейный по компонентам тензора деформаций $u_{\alpha \beta}$ и напряженности электрического поля $E_{\alpha}$ вклад редкоземельных ионов в свободную энергию кристалла может быть представлен в виде

$$
\mathscr{F}_{\text {pe }}=\frac{N}{2} \sum_{q=1,2}\left\langle\mathscr{H}_{\text {eff }}\right\rangle_{q},
$$

где $N$ - число ионов в кристалле. Эффективный гамильтониан $\mathscr{H}_{\text {eff }}$ определен выражением (7), а символ $\langle\ldots\rangle_{q}$ означает температурное усреднение по невозмущенным волновым функциям ионов в кристаллическом и магнитном полях в каждой подрешетке $(q=1,2)$.

При отсутствии магнитного поля (магнитного порядка) усреднение операторов $C_{q}^{k}$, входящих в (7), дает отличный от нуля результат только для оператора $C_{0}^{2}$, поскольку невозмущенные функции при $H_{q}=0$ представляют собой совокупность гармоник, отличающихся по магнитному квантовому числу на \pm 3 . В этом случае уравнение (8) приводится к виду

$$
\begin{aligned}
\mathscr{F}_{\mathrm{pe}}= & -N\left(A_{1}\left(E_{x}\left(u_{x x}-u_{y y}\right)-2 E_{y} u_{x y}\right)\right. \\
& \left.+A_{2}\left(E_{x} u_{y z}-E_{y} u_{x z}\right)\right) \sqrt{\frac{2}{3}}\left\langle C_{0}^{2}\right\rangle .
\end{aligned}
$$

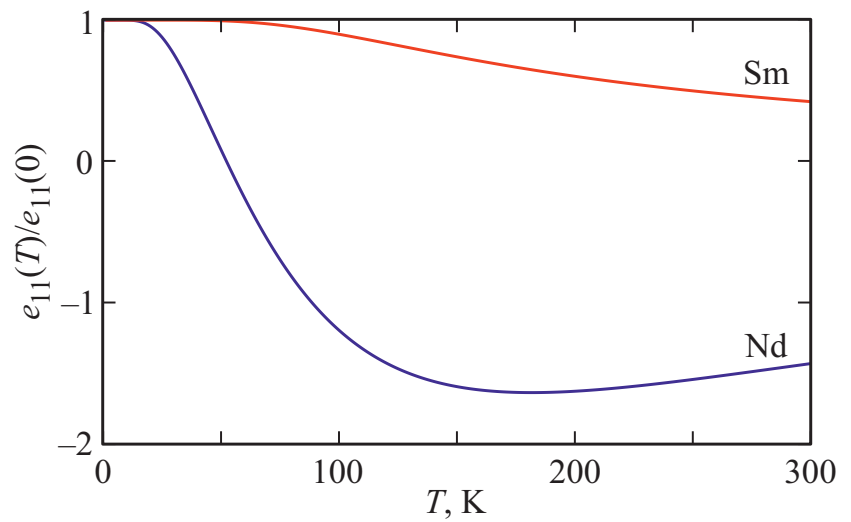

Рис. 1. Графики температурных зависимостей пьезоэлектрического модуля $e_{11}$, выраженного в единицах его значения при $T \rightarrow 0 \mathrm{~K}$, для ферроборатов неодима и самария в нулевом эффективном магнитном поле.

С помощью этого уравнения найдем компоненты вектора электрической поляризации

$$
\begin{gathered}
P_{x}=-\frac{\partial \mathscr{F}_{\mathrm{pe}}}{\partial E_{x}}=N\left(A_{1}\left(u_{x x}-u_{y y}\right)+A_{2} u_{y z}\right) \sqrt{\frac{2}{3}}\left\langle C_{0}^{2}\right\rangle, \\
P_{y}=-\frac{\partial \mathscr{F}_{\mathrm{pe}}}{\partial E_{y}}=N\left(2 A_{1} u_{x y}+A_{2} u_{x z}\right) \sqrt{\frac{2}{3}}\left\langle C_{0}^{2}\right\rangle, \quad P_{z}=0 .
\end{gathered}
$$

Выражения (10) определяют зависимость компонент вектора поляризации ферроборатов при $H_{q}=0$ и $T>T_{N}$ и алюмоборатов от компонент тензора деформации и от температуры. Также из этих выражений могут быть определены пьезоэлектрические модули этих соединений (используем обозначения Фойгта)

$$
e_{11}=N A_{1} \sqrt{\frac{2}{3}}\left\langle C_{0}^{2}\right\rangle, \quad e_{14}=N A_{2} \sqrt{\frac{2}{3}}\left\langle C_{0}^{2}\right\rangle .
$$

Интересно заметить, что зависимости обоих пьезомодулей от температуры для данного соединения описываются одной и той же функцией температуры, но, конечно, разными функциями для различных соединений. На рис. 1 показаны эти температурные зависимости для ферроборатов неодима и самария. Согласно [24], при близких к точке Нееля температуpax $e_{11}=(1.4 \pm 0.3) \mathrm{C} / \mathrm{m}^{2}$ для обоих соединений, а $e_{14}=(0.4 \pm 0.2) \mathrm{C} / \mathrm{m}^{2}$ для ферробората неодима.

\section{4. Магнитостимулированный пьезоэлектрический эфффект}

Из выражений (10) следует, что в нулевом эффективном магнитном поле вектор поляризации кристаллов ферроборатов неодима и самария лежит в базовой плоскости. Отличие компоненты $P_{z}$ от нуля возможно только определенном „магнитном“ состоянии кристалла, 
что непосредственно вытекает из (8). В самом деле, из (8) найдем, что

$$
\begin{aligned}
P_{z}= & -\frac{N}{2} \sum_{q=1,2}\left(A_{1}\left(u_{x x}-u_{y y}\right)+A_{2} u_{y z}\right)\left\langle C_{-1}^{2}-C_{1}^{2}\right\rangle_{q} \\
& -i\left(2 A_{1} u_{x y}+A_{2} u_{x z}\right)\left\langle C_{-1}^{2}+C_{1}^{2}\right\rangle_{q} .
\end{aligned}
$$

Аналогично тому, как из (10) были получены выражения для пьезоэлектрических модулей $e_{11}$ и $e_{14}$, используя формулу (11), запишем выражения для магнитоиндуцированных пьезомодулей

$$
\begin{aligned}
& e_{31}^{m}=-\frac{N A_{1}}{2} \sum_{q=1,2}\left\langle C_{-1}^{2}-C_{1}^{2}\right\rangle_{q}, \\
& e_{34}^{m}=-\frac{N A_{2}}{2} \sum_{q=1,2}\left\langle C_{-1}^{2}-C_{1}^{2}\right\rangle_{q}, \\
& e_{36}^{m}=-\frac{N A_{1}}{2} \sum_{q=1,2} i\left\langle C_{-1}^{2}+C_{1}^{2}\right\rangle_{q}, \\
& e_{35}^{m}=-\frac{N A_{2}}{2} \sum_{q=1,2} i\left\langle C_{-1}^{2}+C_{1}^{2}\right\rangle_{q} .
\end{aligned}
$$

Верхний индекс $m$ подчеркивает тот факт, что возникновение поляризованности кристалла вдоль кристаллографического направления $c$ обуславливается наличием эффективного магнитного поля (1), действующего на редкоземельные ионы.

Для нахождения компоненты $P_{z}$ и пьезомодулей (12) необходимо выполнить температурное усреднение по невозмущенным волновым функциям редкоземельных ионов в кристаллическом и магнитном полях в каждой подрешетке $(q=1,2)$. Гамильтониан $\mathscr{H}_{C F}$ кристаллического поля дается выражением (2), а гамильтониан $\mathscr{H}_{Z}$ редкоземельного иона в магнитном поле можно представить в виде

$$
\mathscr{H}_{Z}=g_{J} \mu_{B} \mathbf{J H}_{\text {eff }}
$$

где $\mathbf{H}_{\text {eff }}-$ эффективное магнитное поле, действующее на редкоземельный ион.

В эффективном магнитном поле основной крамерсовский дублет редкоземельного иона $\left(\left|\psi_{0}\right\rangle,\left|\bar{\psi}_{0}\right\rangle\right)$, приведенный в табл. 1, расщепляется на два близко лежащих уровня, энергии и волновые функции которых для иона $q$-й подрешетки имеют вид [20]:

$$
E_{ \pm}^{(q)}= \pm g_{J} \mu_{B} \Delta\left(H_{q}\right), \quad\left|\psi_{ \pm}^{(q)}\right\rangle=a_{ \pm}^{(q)}\left|\psi_{0}\right\rangle \pm b_{ \pm}^{(q)}\left|\bar{\psi}_{0}\right\rangle,
$$

где $g_{J}-$ множитель Ланде, $\mu_{B}-$ магнетон Бора, функция $\Delta\left(H_{q}\right)$ имеет вид

$$
\Delta\left(H_{q}\right)=\sqrt{g_{z}^{2} H_{q z}^{2}+g_{\perp}^{2}\left(H_{q x}^{2}+H_{q y}^{2}\right)},
$$

Таблица 2. Значения множителя Ланде $g_{J}$, ненулевых компонент $g$-фактора спектроскопического расщепления, полей $f-d$ обмена

\begin{tabular}{c|c|c|c|c}
\hline Ион & $g_{J}$ & $g_{\perp}$ & $g_{z}$ & $H_{\mathrm{mol}}, \mathrm{kOe}$ \\
\hline $\mathrm{Nd}^{3+}$ & $\frac{8}{11}$ & 1.751 & 0.964 & 50 \\
$\mathrm{Sm}^{3+}$ & $\frac{2}{7}$ & 1.441 & 0.379 & 330
\end{tabular}

а коэффициенты $a_{ \pm}^{(q)}$ и $b_{ \pm}^{(q)}$ записываются следующим образом:

$$
\begin{gathered}
a_{ \pm}^{(q)}=\frac{i}{\sqrt{2}}\left(1 \mp \frac{g_{z} H_{q z}}{\Delta\left(H_{q}\right)}\right) e^{i \varphi_{q} / 2}, \\
b_{ \pm}^{(q)}=-\frac{i}{\sqrt{2}}\left(1 \pm \frac{g_{z} H_{q z}}{\Delta\left(H_{q}\right)}\right) e^{-i \varphi_{q} / 2},
\end{gathered}
$$

где фаза $\varphi_{q}$ определяется соотношением $\operatorname{tg} \varphi_{q}=$ $=H_{q y} / H_{q x}$. При записи всех этих выражений учтено, что ненулевыми являются лишь диагональные компоненты тензора $g$-фактора спектроскопического расщепления $g_{x x}=g_{y y}=g_{\perp}$ и $g_{z z}=g_{z}$, см. табл. 2 .

Вклад вышележащих уровней в волновую функцию редкоземельного иона в магнитном поле может быть найден с использованием теории возмущений

$$
\left|\Psi_{ \pm}^{(q)}\right\rangle=\left|\psi_{ \pm}^{(q)}\right\rangle+\sum_{k} \frac{\left\langle\psi_{k}\left|\mathscr{H}_{Z}\right| \psi_{ \pm}^{(q)}\right\rangle}{E_{0}-E_{k}}\left|\psi_{k}\right\rangle .
$$

Оператор $\mathscr{H}_{Z}$ представляет собой зеемановскую часть гамильтониана редкоземельного иона в $q$-й подрешетке, см. уравнение (12). Энергии $E_{k}$ и волновые функции $\left(\left|\psi_{k}\right\rangle,\left|\bar{\psi}_{k}\right\rangle\right)$ крамерсовских дублетов, полученных расщеплением основного мультиплета в кристаллическом поле, приведены в табл. 1. Заметим еще, что выражения (13) и (14) справедливы для не слишком сильных магнитных полей $H \ll E_{k} / \mu_{B} \sim 10^{6} \mathrm{Oe}$.

Усреднения в уравнениях (11) и (12), проведенные согласно процедуре, подробно описанной в работе [20], приводят к следующим результатам:

$$
\begin{gathered}
\left\langle C_{-1}^{2}-C_{1}^{2}\right\rangle_{q}=f_{1} H_{q x} H_{q z} \chi\left(H_{q}, T\right), \\
i\left\langle C_{-1}^{2}+C_{1}^{2}\right\rangle_{q}=f_{2} H_{q y} H_{q z} \chi\left(H_{q}, T\right),
\end{gathered}
$$

где

$$
\chi\left(H_{q}, T\right)=\frac{1}{\Delta\left(H_{q}\right)} \text { th }\left(\frac{g_{J} \mu_{B} \Delta\left(H_{q}\right)}{k T}\right),
$$

a $f_{1}$ и $f_{2}-$ вещественные константы, определяемые волновыми функциями редкоземельного иона в кристаллическом поле.

Теперь потребуется явное выражение для эффективного магнитного поля $\mathbf{H}_{\mathrm{eff}}$ в ферроборате неодима, для его записи воспользуемся сформулированной в работе [25] моделью магнитной структуры этого материала. 

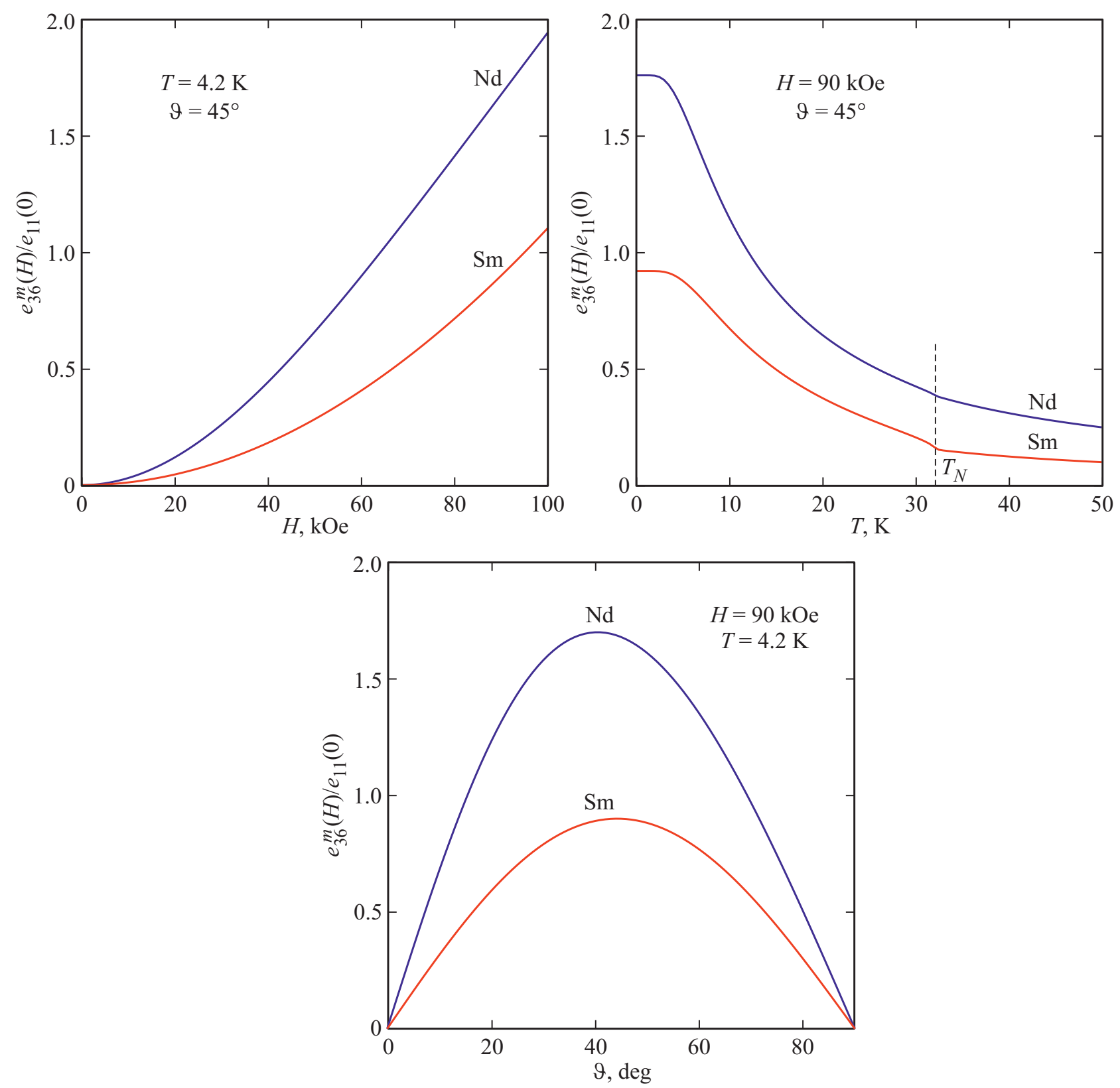

Рис. 2. $a$ : графики полевых зависимостей пьезоэлектрического модуля $e_{36}^{m}$ для ферроборатов неодима и самария, полученных при температуре $T=4.2 \mathrm{~K}, \vartheta=45^{\circ} . b$ : графики температурных зависимостей пьезоэлектрического модуля $e_{36}^{m}$ для ферроборатов неодима и самария, полученных во внешнем поле $H=90 \mathrm{kOe}, \vartheta=45^{\circ} . c$ : графики ориентационных зависимостей пьезоэлектрического модуля $e_{36}^{m}$ для ферроборатов неодима и самария, полученных при температуре $T=4.2 \mathrm{~K}$ во внешнем поле $H=90 \mathrm{kOe}$.

Согласно этой модели, подсистема ионов железа в ферроборате неодима представлена в виде двух магнитных подрешеток, которые связаны обменным полем, формирующим антиферромагнитное упорядочение. Ионы неодима увеличивают стабильность базисной $a b$ плоскости для магнитных моментов ионов железа, поэтому вектор антиферромагнетизма $\mathbf{L}$ лежит в этой плоскости во всем интервале температур вплоть до точки Нееля, образуя, вообще говоря, некоторый угол $\varphi$ с координатной осью $x$ :

$$
\mathbf{e}_{L}=\mathbf{e}_{x} \cos \varphi+\mathbf{e}_{y} \sin \varphi
$$

Здесь введен единичный вектор $\mathbf{e}_{L}$, задающий направление вектора антиферромагнетизма,

$$
\mathbf{e}_{L}=\frac{\mathbf{M}_{1}-\mathbf{M}_{2}}{2 M_{0}},
$$

где $\mathbf{M}_{1,2}$ - магнитные моменты ионов железа в подрешетках 1 и 2, причем $\left|\mathbf{M}_{1,2}\right|=M_{0}$.

В сравнительно сильных магнитных полях (превышающих величину $10 \mathrm{kOe})$ магнитная и доменная структуры кристалла перестраиваются таким образом, что вектор $\mathbf{L}$ становится направленным перпендикулярно внешнему магнитному полю и однородным по всему объему 
образца. При этом процесс намагничивания обуславливается скашиванием подрешеток ионов железа (явление спин-флипа, соответствующее поле $\left.H_{\text {flip }} \sim 1000 \mathrm{kOe}\right)$ и редкоземельной подсистемой. Будем считать, что к кристаллу ферробората приложено внешнее магнитное поле $\mathbf{H}$ таким образом, что $\mathbf{H} \perp \mathbf{L}$, т. е.

$$
\mathbf{H}=H_{z} \mathbf{e}_{z}+H_{\perp}\left[\mathbf{e}_{z} \times \mathbf{e}_{L}\right],
$$

где $H_{z}=H \cos \vartheta, H_{\perp}=H \sin \vartheta$, а $\vartheta-$ угол, образованный внешним магнитным полем Н с координатной осью $z$.

Редкоземельные ионы также разбиваются на две подрешетки, обменно связанные с соответствующими подрешетками ионов железа. Влияние подсистемы железа на редкоземельные ионы адекватно описывается в рамках модели молекулярного поля, причем величина обменного поля составляет $H_{\mathrm{mol}}=50 \mathrm{kOe}$. При этом обменное взаимодействие между подрешетками самих редкоземельных ионов пренебрежимо мало.

С учетом вышесказанного, для подрешеток $q=1,2$ выражение для эффективного магнитного поля $\mathbf{H}_{q}$ записывается в виде

$$
\begin{aligned}
\mathbf{H}_{q}= & (-1)^{q} H_{\mathrm{mol}} \sqrt{1-\left(\frac{H}{H_{\text {flip }}}\right)^{2}} \mathbf{e}_{L} \\
& +\left(H_{z} \mathbf{e}_{z}+H_{\perp}\left[\mathbf{e}_{z} \times \mathbf{e}_{L}\right]\right)\left(1+\frac{H_{\text {mol }}}{H_{\text {flip }}}\right)
\end{aligned}
$$

Отметим, что сходная картина пригодна и для описания магнитной структуры ферробората самария, с той лишь разницей, что величина обменного поля в нем составляет $330 \mathrm{kOe}$.

Из уравнений (16) и (18) ясно, что пьезоэлектрические модули $e_{31}^{m}$ и $e_{34}^{m}$ тождественно равны нулю при любой температуре, а также при любой величине и ориентации внешнего магнитного поля (17). Пьезоэлектрические модули $e_{36}^{m}$ и $e_{35}^{m}$ также равны нулю, если внешнее магнитное поле направлено коллинеарно вектору $\mathbf{e}_{L}$ или вектору $\mathbf{e}_{z}$, в остальных случаях они отличны от нуля. Следует заметить, что нижние индексы рассматриваемых пьезомодулей здесь понимаются как соответствующие направлениям, задаваемых правой тройкой векторов $\left\{\mathbf{e}_{L} ;\left[\mathbf{e}_{z} \times \mathbf{e}_{L}\right] ; \mathbf{e}_{z}\right\}$.

На рис. 2 показаны температурные зависимости пьезоэлектрического модуля $e_{36}^{m}$ при фиксированном значении внешнего магнитного поля $\mathbf{H}$, а также полевые и ориентационные зависимости при фиксированной температуре для ферроборатов неодима и самария. Величины пьезомодулей приведены к значению пьезомодуля $e_{11}$ для каждого из соединений в нулевом эффективном магнитном поле при стремящейся к нулю температуре для удобства сравнения величин пьезоэлектрических эффектов. При фиксированной температуре эти пьезомодули в слабых полях квадратично зависят от величины поля, в более сильных полях зависимость становится линейной. С ростом температуры величины пьезоэлектрических модулей монотонно убывают.

\section{5. Заключение}

В настоящей работе разработана квантовая теория пьезоэлектрического эффекта в редкоземельных ферроборатах. Показано, что за возникновение эффекта отвечают преимущественно одноионные механизмы, что приводит к достаточно большим значениям поляризации, индуцированной в этих материалах при их деформации даже при комнатных температурах (приблизительно на порядок больше, чем в кварце), поэтому редкоземельные ферробораты могут представлять определенный интерес для практического использования. Рассчитаны зависимости пьезоэлектрических модулей ферроборатов неодима и самария от температуры. Указано на возможность возникновения пьезоэлектрического эффекта, обусловленного наличием магнитного порядка в кристалле при температурах ниже точки Нееля, при приложении внешнего магнитного поля, причем имеется возможность управления величинами пьезоэлектрических модулей с помощью внешнего магнитного поля. Несмотря на то, что в работе подробно рассмотрены лишь ферробораты неодима и самария, развитая теория может быть применена с небольшими изменениями не только к другим редкоземельным ферроборатам, но также и к редкоземельным материалам других классов.

\section{Финансирование работы}

Настоящая работа была выполнена при финансовой поддержке Российского фонда фундаментальных исследований (грант № 18-02-00994 и № 19-52-80024).

\section{Конфликт интересов}

Авторы заявляют, что у них нет конфликта интересов.

\section{Список литературы}

[1] J. Curie, P. Curie. Bull. Soc. Minerolog. France 3, 90 (1880).

[2] G. Lippman. Ann. Chem. Phys. 24, 145 (1881).

[3] J. Curie, P. Curie. Compt. Rendus 93, 1137 (1881).

[4] Л.Д. Ландау, Е.М. Лифшиц. Электродинамика сплошных сред. Физматлит, М. (2005). 656 с.

[5] В.А. Головнин, И.А. Каплунов, О.В. Малышкина, Б.Б. Педько, А.А. Мовчикова. Физические основы, методы исследования и практическое применение пьезоматериалов. Техносфера, М. (2013). 273 с.

[6] https://www.acmite.com/market-reports/materials/globalpiezoelectric-device-market.html

[7] S.J. Rupitsch. Piezoelectric sensors and actuators: fundamentals and applications. Springer-Verlag, Berlin Heidelberg (2019). 559 p.

[8] Piezoelectric transducers and applications / Ed. A.A. Vives. Springer-Verlag, Berlin Heidelberg (2008). 532 p.

[9] S.N. Shevtsov, A.N. Soloviev, I.A. Parinov, A.V. Cherpakov, V.A. Chebanenko. Piezoelectric actuators and generators for energy harvesting: research and development. Springer-Verlag, Berlin Heidelberg (2018). 182 p. 
[10] V. Lavrinenko, I. Lavrinenko. Piezoelectric motors: the basic theory, principles of design and realization. Lambert Academic Publishing, Riga (2016). 220 p.

[11] B. Voigtlaender. Scanning probe microscopy: atomic force microscopy and scanning tunneling microscopy. SpringerVerlag, Berlin Heidelberg (2015). 382 p.

[12] K.M. Rabe, P. Ghosez. Topics Appl. Phys. 105, 117 (2007).

[13] A.P. Aslla-Quispe, R.H. Miwa, J.D.S. Guerra. Ferroelectrics 535, 65 (2018).

[14] G. Saghi-Szabo, R.E. Cohen, H. Krakauer. Phys. Rev. B 59, 12771 (1999).

[15] Directive 2002/95/EC of the European Parliament and of the Council of 27 January 2003 on the restriction of the use of certain hazardous substances in electronic equipment. Official J. Eur. Union 37, 19 (2003).

[16] А.К. Звездин, С.С. Кротов, А.М. Кадомцева, Г.П. Воробьев, Ю.Ф. Попов, А.П. Пятаков, Л.Н. Безматерных, Е.Н. Попова. Письма в ЖЭТФ 81, 6, 335 (2005). [A.K. Zvezdin, S.S. Krotov, A.M. Kadomtseva, G.P. Vorobev, Yu.F. Popov, A.P. Pyatakov, L.N. Bezmaternykh, E.A. Popova. JETP. Lett. 81, 272 (2005).]

[17] А.М. Кадомцева, Ю.Ф. Попов, Г.П. Воробьёв, А.П. Пятаков, С.С. Кротов, К.И. Камилов, В.Ю. Иванов, А.А. Мухин, А.К. Звездин, А.М. Кузьменко, Л.Н. Безматерных, И.А. Гудим, В.Л. Темеров. Физика низких температур 36, 6, 640 (2010). [A.M. Kadomtseva, Yu.F. Popov, G.P. Vorobev, A.P. Pyatakov, S.S. Krotov, K.I.A. Gudim, V.L. Temerov. Low Temp. Phys. 36, 511 (2010).]

[18] A.M. Kadomtseva, Yu.F. Popov, G.P. Vorobev, N.V. Kostyuchenko, A.I. Popov, A.A. Mukhin, V.Yu. Ivanov, L.N. Bezmaternykh, I.A. Gudim, V.L. Temerov, A.P. Pyatakov, A.K. Zvezdin. Phys. Rev. B 89, 014418 (2014).

[19] В.Ю. Иванов, А.М. Кузьменко, А.А. Мухин. Письма в ЖЭТФ 105, 7, 430 (2017). [V.Y. Ivanov, A.M. Kuzmenko, A.A. Mukhin. JETP Lett. 105, 435 (2017).]

[20] A.I. Popov, D.I. Plokhov, A.K. Zvezdin. Phys. Rev. B 87, 024413 (2013).

[21] M.N. Popova, E.P. Chukalina, T.N. Stanislavchuk, B.Z. Malkin, A.R. Zakirov, E. Antic-Fidancev, E.A. Popova, L.N. Bezmaternykh, V.L. Temerov. Phys. Rev. B 75, 224435 (2007).

[22] M.N. Popova, T.N. Stanislavchuk, B.Z. Malkin, L.N. Bezmaternykh. Phys. Rev. B 80, 195101 (2009).

[23] Н.Ф. Ведерников, А.К. Звездин, Р.З. Левитин, А.И. Попов. ЖЭТФ 93, 6, 2161 (1987).

[24] Т.Н. Гайдамак, И.А. Гудим, Г.А. Звягина, И.В. Билыч, Н.Г. Бурма, К.Р. Жеков, В.Д. Филь. Физика низких температур 41, 8, 792 (2015).

[25] T.N. Gaydamak, I.A. Gudim, G.A. Zvyagina, I.V. Bilych, N.G. Burma, K.R. Zhekov, V.D. Fil. Phys. Rev. B 92, 214428 (2015).

[26] А.К. Звездин, Г.П. Воробьёв, Ю.Ф. Попов, А.П. Пятаков, Л.Н. Безматерных, А.В. Кувардин, Е.А. Попова. Письма в ЖЭТФ 83, 11, 600 (2006).

Редактор Т.Н. Василевская 